Contenidos digitales

para niños
Contenidos digitales para niños | Revista Virtual

Universidad Católica del Norte, 64, 119-150

ISSN: 0124-5821 (En línea)

Amador-Baquiro, J. C. (septiembre-diciembre, 2021). Contenidos digitales para niños de primera infancia: el caso del Portal Maguaré. Revista Virtual Universidad Católica del Norte,

(64), 119-150. https://www.doi.org/10.35575/rvucn.n64a6

\title{
Contenidos digitales para niños de primera infancia: el caso del portal Maguaré1
}

\section{Digital content for early childhood children: Maguaré portal case}

\author{
Juan Carlos Amador-Baquiro \\ Doctor en Educación \\ Facultad de Ciencias y Educación Universidad Distrital Francisco José de Caldas \\ Bogotá, Colombia \\ jcamadorb@udistrital.edu.co \\ Orcid: http://orcid.org/0000-0002-5575-1755 \\ CvLac: https://scienti.minciencias.gov.co/cvlac/visualizador/generarCurriculoCv.do?cod_rh=0000668923
}

Recibido: 05 de julio de 2020

Evaluado: 01 de febrero de 2021

Aprobado: 13 de abril de 2021

Tipo de artículo: Investigación Científica y Tecnológica

\section{Resumen}

El portal digital Maguaré, implementado por el Ministerio de Cultura de Colombia, tiene como propósito ofrecer experiencias de inmersión a los niños de primera infancia para que interactúen con contenidos digitales, relacionados con el patrimonio cultural de la Nación. A partir de una perspectiva cultural de la comunicación digital y de una mirada crítica de la primera infancia, comprendida como una construcción social que se redefine en la contemporaneidad tras la mediatización de la realidad, el artículo explora, desde una perspectiva cualitativa, algunos contenidos de este portal, empleando como metodología el análisis crítico del discurso multimodal,

\footnotetext{
${ }^{1}$ Este artículo surge de la investigación titulada: Usos y apropiaciones de niños y niñas de primera infancia en el portal Maguaré, desarrollada en los años 2017 y 2019, a partir del acta de cooperación firmada entre el Ministerio de Cultura de Colombia y el Doctorado Interinstitucional en Educación de la Universidad Distrital Francisco José de Caldas 2018-05-20. En adelante, el término niño y niños también incluye a la niña y a las niñas.
} 


\section{Contenidos digitales}

para niños
Contenidos digitales para niños | Revista Virtual

Universidad Católica del Norte, 64, 119-150

ISSN: 0124-5821 (En línea)

el cual analiza las funciones comunicativas de los diversos modos semióticos que configuran las narrativas en la actualidad. En los resultados sobresale la complementariedad entre distintos modos semióticos en los contenidos, situación que propicia la significación y la sensibilidad, pero también los estereotipos. Como conclusiones se destacan: el énfasis en el modo verbal, así como su integración con la imagen y la codificación textual, hacen posible que el usuario apropie referentes que representan la diversidad cultural, étnica y territorial; además, Maguaré emplea estrategias de racialización que anclan a las personas afrodescendientes, representadas en una diferencia subalternizada, inventada y narrada desde un mundo imaginado blanco-mestizo.

Palabras clave: Comunicación digital interactiva; Contenidos digitales; Discurso multimodal; Primera infancia.

\begin{abstract}
Maguare portal, implemented by the Colombian Ministry of Culture, offers immersion experiences for early childhood children for interact with content related to the cultural heritage of the nation. The article uses a cultural perspective of interactive digital communication and a critical view of early childhood, understood as a social construction that is redefined in contemporary society after the mediation of reality. The article explores from a qualitative perspective some contents of this portal using the critical analysis of multimodal discourse, which analyzes the communicative functions of the various semiotic mode that configure narratives today. In the results, the complementarity between different semiotic modes in the contents favors significance and sensitivity, but also stereotypes. As conclusions, the article exposes: the emphasis on the verbal mode of the contents, as well as their integration with the image and the textual coding, make it possible for the user to access, browse and approve related considerations that represent diversity cultural, ethnic and territorial of Colombia; and Maguare used racialization strategies that anchor Afro-descendants represented in a subalternized and marginal difference, invented and narrated from an ideal white-mestizo imagined world.
\end{abstract}

Keywords: Interactive digital communication; Digital contents; Multimodal discourse; Early childhood. 
Contenidos digitales

para niños
Contenidos digitales para niños | Revista Virtual

Universidad Católica del Norte, 64, 119-150

ISSN: 0124-5821 (En línea)

\section{Introducción}

Desde inicios del siglo XXI, en varios países se ha discutido la conveniencia o no de diseñar e implementar contenidos digitales para los niños de primera infancia. Para algunos investigadores (Buckingham, 2012; Chaudron et al., 2015; Kelly, 2016), el acceso intuitivo o guiado a este tipo de contenidos genera profundos interrogantes, en torno a los aportes o perjuicios de estas representaciones multimodales en el desarrollo integral de los niños. Por otro lado, algunos estudios (Benítez, 2013; Corredor y Garzón, 2015; Rodríguez Illera et al. 2004) problematizan las influencias de estas iniciativas digitales en el despliegue de la educación infantil a partir del arte, la literatura, el lenguaje y las matemáticas. Asimismo, se han interrogado los discursos educativos, comunicacionales y estéticos empleados por los creadores de estos contenidos, tanto en los procesos de producción como de difusión. Como se explicará más adelante, de acuerdo con Kress \& Van Leeuwen (2001), la composición de determinado contenido multimodal puede propiciar ciertas orientaciones cognitivas e ideológicas sobre la realidad social y cultural en los usuarios. Por estas razones, parece necesario profundizar en el análisis crítico de aquellas representaciones sobre la diversidad y la diferencia, que son tramitadas por medio de sitios web, dirigidos hacia la primera infancia.

De acuerdo con estas consideraciones iniciales, en este estudio se adoptó como unidad de análisis el portal Maguaré, un sitio web del Ministerio de Cultura de Colombia, el cual busca que los niños de primera infancia, en calidad de usuarios, accedan a los bienes culturales de la Nación. Específicamente, Maguaré es un universo narrativo que posibilita la inmersión y la interactividad de sus usuarios, a partir de cuatro escenarios digitales: música (Caja de Música), literatura (Teatro de la Imaginación), tradición oral (Palabras al aire) y exploración del cuerpo (Mi Casa en el Árbol). En Maguaré se encuentran contenidos textuales, visuales, sonoros, audiovisuales y de animación digital, tales como rondas, juegos, cuentos, libros, canciones, videos musicales, series animadas y aplicaciones móviles. El portal cuenta con una navegación aleatoria que hace posible al usuario explorar los contenidos de diversas formas. Al parecer, estos atributos intensifican los grados de interactividad, en la medida que los usuarios lo exploren con frecuencia, y se enmarcan en una 
Contenidos digitales

para niños
Contenidos digitales para niños | Revista Virtual

Universidad Católica del Norte, 64, 119-150

ISSN: 0124-5821 (En línea)

nueva modalidad de consumo simbólico (Buckingham, 2012; Johansson, 2010; Livingstone, 2006).

En el ámbito internacional, desde 2013, la Coalición Internacional de Calificación por Edad (IARC) se encarga de clasificar y calificar, según las edades de las personas, los juegos y las aplicaciones digitales que se lanzan al mercado, con el fin de garantizar que los usuarios cuenten con la seguridad y la accesibilidad suficientes en las distintas plataformas. A estas regulaciones, se someten tiendas como Google Play, Nintendo, E-shop y Windows Store (IARC, s.f.) e iniciativas de educación virtual. Por su parte, teniendo en cuenta la creciente oferta de servicios digitales para niños en general, y recientemente la circulación de contenidos para niños de primera infancia, UNICEF (como se citó en Galvis, 2014) encuentra que estos lenguajes digitales son una realidad del mundo contemporáneo, que pueden ofrecer oportunidades importantes de sano entretenimiento, aunque también convertirse en posibilidad para el desarrollo de la innovación educativa y la formación del talento humano.

En sintonía con lo anterior, en varios países se han diseñado e implementado portales culturales y educativos dirigidos a niños de primera infancia. Se destacan tres experiencias en el ámbito internacional cuyos contenidos son únicamente para estas personas o para los docentes de educación inicial. La primera, originada en España, llamada Educarex, pretende mejorar la interacción de estos niños con las tecnologías de la información y la comunicación -TIC- y ofrecer recursos de apoyo a la educación infantil. La segunda, llamada Yoopa, que nace en Canadá en el año 2001, es un portal con juegos, páginas para colorear, videos y una sección llamada El Universo de Theo, en la que el usuario puede ser parte de un juego de rol como periodista. El portal ofrece un espacio para padres con información nutricional, vida familiar y educación. Por último, desde el Ministerio de Educación y Deporte de Argentina, fue creado Pakapaka, un portal para niños de primera infancia que ofrece acceso a contenidos culturales y educativos para su desarrollo integral, desde una perspectiva de derechos.

En el ámbito nacional, además de Maguaré, se encuentran otros portales dirigidos a población infantil con distintos propósitos y alcances. A modo de ejemplo, se encuentra el sitio web Música Libre, una iniciativa digital de la Corporación Cultural de proyección 
Contenidos digitales

para niños
Contenidos digitales para niños | Revista Virtual

Universidad Católica del Norte, 64, 119-150

ISSN: 0124-5821 (En línea)

artística Cantoalegre de Medellín, la cual ofrece a sus usuarios entretenimiento y aprendizaje a través de secciones como descargas (canciones e imágenes), radio (música online y descargable), cantar y jugar (videos, karaokes y cuentos), y canta y enseña (talleres y artículos con secuencias didácticas). También, se encuentra el portal CuidArte, un sitio Web con herramientas para que los padres y cuidadores conozcan sobre pautas de crianza y cuidado para la primera infancia, a partir de cuatro secciones: Aconteceres, CuidArte, Biblioteca y Aliados. Por último, desde el Instituto Distrital de las Artes (IDARTES) de Bogotá, se diseñó e implementó el portal Creciendo con Kike, que surge a partir de un proyecto del Gobierno distrital, en el que varios niños menores de 6 años participaron de la mano de 300 artistas en su creación y adecuación. El portal contiene las secciones juegos, audiovisuales y música, así como una serie de enlaces al sitio web Tejedores de vida arte en primera infancia.

Por otra parte, las investigaciones académicas dedicadas a problematizar la relación infancia - comunicación digital - contenidos digitales, se pueden dividir en dos grandes tendencias: diseño de contenidos digitales para estos niños, y medios digitales en la educación infantil. En relación con la primera tendencia, se destacan los trabajos de Lizarazo (2012), Chaudron et al. (2015), Paudel et al. (2017) y Rodríguez Illera et al. (2004), quienes desarrollan investigaciones empíricas a partir del diseño, implementación y validación de contenidos digitales alojados en portales, sitios web, material multimedia, hipermedias y micrositios, con niños de varias edades, incluyendo primera infancia. Mientras que algunos analizan los aspectos estéticos, comunicativos y educativos de las interfaces y los contenidos, haciendo énfasis en la composición de estos, los criterios de accesibilidad y navegabilidad, así como sus atributos interactivos (Chaudron et al., 2015; Lizarazo, 2012; Paudel et al., 2017), otros hacen énfasis en los aportes de estos diseños digitales en los procesos de lectura y escritura, el aprendizaje autónomo, el desarrollo sociolingüístico y la inteligencia de los usuarios (Arango, 2014; Rodríguez Illera et al., 2004). Otros trabajos, como los de Kelly (2016) y Vallejo (2001), se centran en el análisis del contenido de distintos sitios web, orientados a niños de primera infancia, e interrogan la función mediadora de estas tecnologías en la producción cultural y las interacciones sociales.

En relación con la segunda tendencia, se encuentran investigaciones que abordan los aportes u obstáculos de los recursos digitales, y en general de los medios digitales, la inclusión digital y el desarrollo de procesos educativos, pedagógicos y/o de aprendizaje, especialmente en 
Contenidos digitales

para niños
Contenidos digitales para niños | Revista Virtual

Universidad Católica del Norte, 64, 119-150

ISSN: 0124-5821 (En línea)

las áreas de artes y lenguaje, por parte de los docentes de educación infantil (Colectivo Educación Infantil y TIC - Universidad del Norte, s.f.; Hernández, 2011; Ramos y González, 2012; Thomson et al., 2014; Giraldo-Vargas, 2011). En estos trabajos se destacan tres perspectivas: el análisis de los posibles cambios en los procesos de enseñanza, por parte de los docentes, al usar recursos digitales para primera infancia; las capacidades de los docentes al emplear recursos digitales, conforme a situaciones diferenciales de los niños (género, etnia, edad, discapacidad); y el potencial didáctico de los medios y recursos digitales, los cuales, a partir de mediaciones específicas como el software libre, los blogs, los cuentos interactivos, las aplicaciones y los videojuegos, contribuyen a alcanzar logros en los aprendizajes de los niños de primera infancia, e incluso al desarrollo de competencias.

Asimismo, esta segunda tendencia incluye perspectivas que se centran en los procesos, prácticas e interacciones de los niños en contextos de educación formal y/o informal. Por ejemplo, Hayes (2010), a partir de investigaciones empíricas con niños, evidencia cómo algunos contenidos digitales en música no se adecúan a sus necesidades e intereses, por lo que recomienda diseñar propuestas interactivas de interpretación y de creación. Por su parte, Giráldez (2013) y Corredor y Garzón (2015) hacen énfasis en la necesidad de propiciar actividades en línea que desarrollen la autonomía y el pensamiento crítico en los niños, acompañados del control parental, que equilibren la implementación de actividades pedagógicas con tecnologías digitales y sin estas. Asimismo, SolomonMoore et al. (2018) analizan los desafíos de los padres de familia frente a las prácticas de sus hijos en los entornos multipantalla de sus hogares. Por último, Benítez (2013) y Romero (2012) coinciden en que el uso de tecnologías digitales en la educación infantil puede potenciar en los niños capacidades para alcanzar procesos de apropiación con el fin de resolver problemas. Particularmente, Romero (2012) encuentra en la televisión para niños un elemento central que aporta a la discusión sobre asuntos propios del desarrollo curricular, la educación psicosocial y la socialización de experiencias en torno a la relación educación - medios digitales - industrias culturales.

El anterior panorama, el cual evidencia la proliferación de portales educativosculturales para niños de primera infancia, así como el interés por desarrollar contenidos y 
Contenidos digitales

para niños
Contenidos digitales para niños | Revista Virtual

Universidad Católica del Norte, 64, 119-150

ISSN: 0124-5821 (En línea)

herramientas digitales, con el fin de optimizar los procesos pedagógicos, comunicativos y estéticos de la educación infantil, desde inicios del siglo XXI en varios países, a partir del Estado e iniciativas privadas, plantea tres tipos de interrogantes. En primer lugar, genera inquietudes frente a las funciones de la comunicación digital interactiva en la potenciación o no del desarrollo de los niños de primera infancia. En segundo lugar, sugiere preguntas frente a los discursos educativos, comunicacionales y estéticos empleados por los creadores para la producción y difusión de contenidos digitales dirigidos a estos niños. Y, en tercer lugar, dado que particularmente Maguaré pretende ofrecer contenidos alusivos al patrimonio cultural colombiano, parece necesario conocer la relación entre los discursos multimodales de estos contenidos y el régimen de la mirada, producido a partir del interés de los creadores y gestores del portal por configurar representaciones en torno a la identidad, el territorio y la diversidad cultural.

De acuerdo con las anteriores consideraciones, el estudio se orientó por la pregunta de investigación: ¿Qué discursos multimodales constituyen los contenidos digitales del portal Maguaré del Ministerio de Cultura de Colombia, dirigidos a niños de primera infancia?

\section{Marco teórico}

\section{Comunicación digital interactiva}

El concepto de comunicación digital interactiva (en adelante CDI) fue acuñado por Scolari (2008) en los inicios del siglo XXI, para mostrar tres particularidades de la comunicación, a través de medios y tecnologías digitales en la posmodernidad. En primer lugar, la necesidad de producir una teoría de la comunicación que explique los atributos del lenguaje digital, la reconfiguración de los medios de comunicación y la emergencia de prácticas sociales en las que los consumidores modifican sus formas de interacción con los humanos, los lenguajes y las máquinas. En segundo lugar, la importancia de analizar la interactividad, dado que se trata de un proceso de interacción potenciado a partir de los medios y las tecnologías digitales. Por último, la CDI plantea la necesidad de transitar no solo de los medios a las mediaciones, tal como lo afirmó Martín-Barbero (2003), en la década de 1980, sino de migrar de las mediaciones a las hipermediaciones. Estas últimas se entienden como "procesos de intercambio, producción y consumo simbólico que se desarrollan en 
Contenidos digitales

para niños
Contenidos digitales para niños | Revista Virtual

Universidad Católica del Norte, 64, 119-150

ISSN: 0124-5821 (En línea)

un entorno caracterizado por una gran cantidad de sujetos, medios y lenguajes interconectados tecnológicamente de manera reticular entre sí" (Scolari, 2008, p. 113).

Las hipermediaciones se constituyen a partir de la integración de los medios de comunicación y los géneros convencionales, luego de ser mediatizados gracias a la digitalización de textos, sonido, imagen estática/animación y linealidad narrativa (Manovich, 2001). Este proceso de "traducción simbólica", al decir de Lévy (2007), hace posible la producción, distribución y consumo de hipertextos, multimedias y narrativas transmediales (Barragán \& Amador, 2020; Díez- Gutiérrez \& Díaz-Nafría, 2018). Estas nuevas textualidades no solo modifican las formas de leer la realidad y de escribir textos complejos, sino también de producir significados a partir de la relación de los agentes sociales con los entornos digitales, los cuales también hacen posible percibir el tiempo y el espacio de formas diferentes. Estos cambios que ocurren en lo virtual afectan la realidad social, simbólica y material, y, al parecer, modifican radicalmente los procesos de participación, interacción, producción y consumo en las sociedades contemporáneas (Gardner \& Davis, 2014).

Por su parte, la interactividad es un proceso de potenciación de las interacciones de los actores sociales, a partir de dos tipos de capacidades humanas: las capacidades selectivointerpretativas, las cuales le permiten al usuario construir sus propias trayectorias de lectura; y las capacidades comunicativas, que intensifican los intercambios y diálogos, tanto de manera sincrónica como asincrónica. A esto añade Lévy (2007), en el ámbito de la cibercultura, que los usuarios pueden experimentar mayores grados de interactividad dependiendo de su capacidad de "control" sobre el dispositivo y la interfaz, lo cual puede favorecer la apropiación y la participación. Por último, de acuerdo con Aparici y Silva (2012), la interactividad, comprendida como forma de participación de los usuarios, cuenta con tres atributos: la participación-intervención, proceso que hace posible al usuario no solo actuar sobre recursos digitales predeterminados, sino intervenir y hasta modificar los contenidos disponibles; la bidireccionalidad-hibridación, la cual comprende procesos de co-creación en los que se imbrican operaciones de codificación y decodificación; y la potencialidad-permutabilidad, que asume la comunicación como un proceso que se configura mediante diversas redes asociativas de conexiones. 


\section{Contenidos digitales para niños de primera infancia}

Más allá de asumirla como grupo etario o etapa de desarrollo, la primera infancia es una construcción social que, en este caso, refiere al conjunto de discursos que pretende prescribir las condiciones del momento inicial de la vida de los seres humanos, específicamente al rango de edad que comprende los cero y los seis años, el cual, parece, constituye el punto de partida para alcanzar el desarrollo, la felicidad y la sociabilidad a lo largo de la vida (Chen et al., 2016; Llobet, 2014). Dado que se trata de un proceso de encuentro, interrelación e intercambio de esa persona recién llegada al mundo con otros seres humanos y no humanos, con determinadas dinámicas del orden social, con particulares referentes socio-culturales, en frecuente tensión entre la reproducción y la transformación, así como con espacios y tiempos construidos socialmente que configuran su subjetividad, existe un consenso global construido a lo largo de los últimos veinte años que plantea la primera infancia como prioridad en las agendas de la política pública de los países, en materia de salud, educación y bienestar social (Mustard, 2003). De acuerdo con la Convención de los Derechos del Niño (1989), la Conferencia Mundial de Educación para Todos en Jomtien (1990), el Foro Mundial sobre la Educación en Dakar (2000) y el Simposio Mundial de Educación Parvularia en Santiago de Chile (2000), entre otros espacios dedicados a prescribir qué es y cómo debe ser la primera infancia, se han producido tres tipos de discursos: el de los derechos, el del desarrollo y el de la inversión ${ }^{2}$.

Asuntos como la crianza, la socialización y la educación de los niños más pequeños ya no dependen exclusivamente de los padres, sino también del Estado y otros agentes sociales. Esta situación evidencia cómo, a la par, la sociedad adultocéntrica pone en juego disputas por el sentido,

\footnotetext{
${ }^{2}$ El discurso de los derechos se originó en las orientaciones de la Convención de los Derechos del Niño (CDN) y su fundamento es la doctrina de la protección integral, así como el carácter prevalente de los derechos de los niños. El discurso del desarrollo, basado en teorías psico-biológicas y psicodinámicas del desarrollo infantil, plantea el ideal del desarrollo del niño a partir de interrelaciones entre procesos sensoriales, expresivos, emocionales, corporales y cognitivos. De acuerdo con las neurociencias, desde la gestación hasta antes de los 5 años se desarrolla el 90 por ciento de las conexiones neuronales del cerebro humano, proceso que determina operaciones mentales relacionadas con el aprendizaje, la concentración, la solución de problemas y la toma de decisiones. Por último, el discurso de la inversión, el cual procede de la economía, plantea la inversión de recursos en la primera infancia es la base del capital humano de un país, y que ésta puede alcanzar hasta tres veces su retorno en menos de tres décadas.
} 
Contenidos digitales

para niños
Contenidos digitales para niños | Revista Virtual

Universidad Católica del Norte, 64, 119-150

ISSN: 0124-5821 (En línea)

que involucran ideales distintos sobre los derechos, el desarrollo, el bienestar y el progreso, y que resultan problemáticos frente a la producción de subjetividades en el mundo capitalista-informacional (Buckingham \& Tingstad, 2010). En este contexto, surge la necesidad de preguntar por otras condiciones sociales, políticas y culturales en las que se tramita la primera infancia, tales como la emergencia de la cultura digital, la reconfiguración del capitalismo a partir de la valorización de la información y el conocimiento, el marcado crecimiento del mercado simbólico, la consolidación de otro modelo de comunicación, impulsado por las industrias culturales y la aparición de las llamadas culturas infantiles (Amador-Baquiro, 2018; Buckingham, 2012; Coenen et al., 2015; Livingstone, 2006).

Al respecto, es importante tener en cuenta que tanto la infancia como los niños en las sociedades contemporáneas están cada vez más condicionados por los medios digitales y sus mercancías asociadas. Las prácticas de uso, apropiación y consumo de medios digitales, por parte de los niños, son frecuentes e intensas, proceso que se inscribe no solo en la mercantilización de la cultura, sino también en el consumo infantil (Buckingham, 2012). Mientras que el consumo infantil de medios y contenidos digitales se hace cada vez más individual, se amplían las relaciones interpersonales, como consecuencia de la interactividad a través de estos medios digitales, se profundiza el uso de dispositivos individuales y emergen las llamadas culturas de dormitorio (Livingstone, 2006; RungePeña, 2016). Estas expresiones de las nuevas culturas infantiles están relacionadas con cambios políticos (nuevas alianzas entre Estado y mercado), cambios económicos (capitalismo informacional y cognitivo) y cambios sociales (nuevas dinámicas en la vida familiar y en las relaciones intergeneracionales).

Luego de este recorrido es posible considerar el consumo infantil como una práctica social y cultural, la cual, quizás, tuvo sus inicios desde el momento en el que se objetivó la infancia como hecho social en la temprana modernidad. No obstante, en el escenario contemporáneo, en el que los procesos de socialización, la sociabilidad, la sensibilidad y el acceso al conocimiento están siendo reconfigurados tras la informatización y mediatización de la cultura (Barragán \& Amador, 2020; Winocur, 2009), es claro que surgen otras prácticas y otras gramáticas del consumo en general, y del consumo infantil en particular. 
Contenidos digitales

para niños
Contenidos digitales para niños | Revista Virtual

Universidad Católica del Norte, 64, 119-150

ISSN: 0124-5821 (En línea)

Por último, de acuerdo con Buckingham (2012), es necesario estudiar el consumo infantil (prácticas y gramáticas) de manera contextual, al menos en su relación con la familia, los pares, la escuela y los medios de comunicación.

\section{Metodología}

Teniendo en cuenta que la investigación buscó analizar los discursos multimodales que constituyen los contenidos del portal Maguaré, se procedió con una estrategia metodológica que empleó el enfoque cualitativo (Vasilachis, 2007) y herramientas del análisis crítico del discurso multimodal (ACDMM) (Kress \& Van Leewen, 2001). Aunque la multimodalidad también se conoce como categoría socio-semiótica, en este trabajo el ACDMM se asume como un método que permite analizar fuentes sonoras, visuales, audiovisuales y/o digitales, portadoras de distintos modos semióticos, que cumplen funciones fundamentales en los procesos de producción, distribución y apropiación de la información, el conocimiento y el significado. Este método ofrece alternativas al estudio de discursos y narrativas constituidos por lenguaje alfabético, imágenes, sonoridades y textos hipermediales que son emitidos a través de interfaces pantallizadas y con tendencia a la interactividad.

Para tal efecto, el ACDMM propone el análisis de cuatro aspectos constitutivos de este tipo de fuentes: el discurso, que refiere al conjunto de conocimientos (expresados en interpretaciones, juicios y argumentos) socialmente construidos, procedentes de algún aspecto de la realidad, producidos en contextos sociales específicos; el diseño, que comprende modos de expresión creados para que un público determinado pueda entender los productos culturales en el contexto de una situación comunicativa dada; la producción, que alude a los contenidos puestos en escena, a partir de una intención comunicativa y performativa; y la distribución, entendida como el potencial de recodificación de los productos, el cual tiene propósitos de registro y/o distribución. Estos aspectos deben ser analizados a partir de los modos semióticos verbal, lingüístico, visual y sonoro.

Para el análisis, se escogió un corpus de 35 contenidos multimodales, correspondientes a las salas digitales ubicadas en el portal. Se definieron tres criterios para la selección y análisis de estos contenidos: el carácter multimodal de la composición del contenido (modos visual, verbal, sonoro y eventualmente lingüístico); que su temática aludiera a la diversidad cultural; y que el 
contenido fuera accesible para el usuario. Por cada contenido se diligenció una ficha de análisis multimodal, la cual se estructuró a partir de los cuatro modos semióticos ya mencionados y de los estratos de interpretación del discurso multimodal propuestos por Kress \& Van Leewen (2001): discurso, diseño, producción y distribución. Posteriormente, se procedió a diligenciar una matriz de triangulación de datos que permitió relacionar los tópicos y las categorías emergentes con algunas perspectivas identificadas, tanto en el estado del arte como en el marco teórico. Tanto en el proceso de sistematización y análisis como en la escritura de los resultados se tuvieron en cuenta criterios éticos para no afectar la privacidad de los usuarios, la seguridad del portal digital y el respeto por los derechos de los niños. En la tabla 1 se presentan los aspectos operativos de la estrategia metodológica.

\section{Tabla 1}

Criterios de la metodología basada en el análisis crítico del discurso multimodal

\begin{tabular}{|c|c|c|c|c|c|}
\hline \multicolumn{6}{|c|}{ Objetivo: Analizar los discursos multimodales que constituyen los contenidos del portal Maguaré } \\
\hline & \multicolumn{4}{|c|}{ Contenidos del portal Maguaré } & Técnicas \\
\hline $\begin{array}{l}\text { Aspectos } \\
\text { del ADMM }\end{array}$ & Caja de música & Palabras al aire & $\begin{array}{l}\text { Sala Mi casa } \\
\text { en el árbol }\end{array}$ & $\begin{array}{c}\text { Sala } \\
\text { Teatro de la } \\
\text { imaginación }\end{array}$ & \multirow[b]{2}{*}{$\begin{array}{l}\text { Diligenciamiento } \\
\text { de ficha de ADMM } \\
\text { por cada contenido }\end{array}$} \\
\hline $\begin{array}{l}\text { Discurso } \\
\text { (verbal, }\end{array}$ & Camusi camu & & & $\begin{array}{l}\text { Cuento } \\
\text { contigo }\end{array}$ & \\
\hline linguiístico, & & & Rompecabezas & Crónicas & \\
\hline $\begin{array}{l}\text { Visual y } \\
\text { sonoro) }\end{array}$ & $\begin{array}{c}\text { Constelaciones } \\
\text { sonoras }\end{array}$ & & Pinta pinta & elefantiles & $\begin{array}{l}\text { Identificación de } \\
\text { tópicos emergentes }\end{array}$ \\
\hline $\begin{array}{l}\text { Diseño (verbal, } \\
\text { lingüístico, }\end{array}$ & Canciones & Sana que sana & $\begin{array}{l}\text { Pipo mi amigo } \\
\text { imaginario }\end{array}$ & $\begin{array}{c}\text { Cuentos } \\
\text { interactivos }\end{array}$ & $\begin{array}{c}\text { atendiendo a los } 4 \\
\text { estratos del ADMM } \\
\text { por ficha }\end{array}$ \\
\hline $\begin{array}{l}\text { visual y } \\
\text { sonoro) }\end{array}$ & Videos musicales & Trabalenguas & Juegos & $\begin{array}{l}\text { Cuentos } \\
\text { narrados }\end{array}$ & $\begin{array}{l}\text { Integración de } \\
\text { tópicos emeroentes }\end{array}$ \\
\hline $\begin{array}{l}\text { Producción } \\
\text { (verbal, }\end{array}$ & $\begin{array}{l}\text { Juega con } \\
\text { canciones }\end{array}$ & Adivinanzas & Chigüiro & Máquina de & $\begin{array}{c}\text { para identificar } \\
\text { categorías }\end{array}$ \\
\hline lingüístico, & Karaoke & Arrullos & Carrusel & & emergentes por \\
\hline $\begin{array}{l}\text { visual y } \\
\text { sonoro) }\end{array}$ & Baila con Guille & Cantos & $\begin{array}{l}\text { Guillermina y } \\
\text { Candelario }\end{array}$ & $\begin{array}{l}\text { Aventuras } \\
\text { animadas }\end{array}$ & $\begin{array}{c}\text { medıo de matrız de } \\
\text { análisis }\end{array}$ \\
\hline $\begin{array}{l}\text { Distribución } \\
\text { (verbal, } \\
\text { lingüístico, }\end{array}$ & $\begin{array}{l}\text { Baile con arma - } \\
\text { DJ }\end{array}$ & & Ana pirata & $\begin{array}{l}\text { Arma tu } \\
\text { cuento }\end{array}$ & $\begin{array}{c}\text { Análisis sistemático } \\
\text { y escritura de } \\
\text { hallazgos }\end{array}$ \\
\hline $\begin{array}{l}\text { visual y } \\
\text { sonoro) }\end{array}$ & & & & $\begin{array}{l}\text { Cuentos al } \\
\text { oído }\end{array}$ & \\
\hline
\end{tabular}

Nota: Elaboración propia. 
En la tabla 1 se explicitan los contenidos analizados por cada una de las salas del portal. Cada uno de estos fue analizado a partir de los estratos del ACDMM ya explicados. Luego del diligenciamiento de la ficha de ADMM por cada contenido, se procedió a identificar los tópicos emergentes. Posteriormente, se integraron los tópicos emergentes, con el fin de conformar campos semánticos, los cuales permitieron construir las categorías inductivas por medio de una matriz de análisis. Por último, se procedió con el análisis sistemático y la escritura de los hallazgos.

\section{Resultados}

Como se mencionó al inicio, Maguaré es un portal interactivo conformado por cuatro salas a las que se puede acceder de manera intuitiva o selectiva. A la vez, cada una de estas salas posee contenidos que se caracterizan por una diversidad de temáticas que emplean distintas técnicas de diseño, a varias apuestas estéticas y comunicativas, así como variadas formas de interactividad. La primera sala, denominada "Caja de música", expone expresiones musicales y sonoridades a través de instrumentos musicales, objetos y melodías. Algunos audios y videoclips hacen referencia a ritmos originarios de algunas regiones de Colombia. La segunda sala, llamada "Mi casa en el árbol”, está conformada de herramientas interactivas como la pintura (análogas al programa paint) y los rompecabezas para potenciar habilidades de observación, identificación, comparación, clasificación y composición en los participantes. Se cuentan historias sobre temáticas infantiles, entre ellas, algunas que promueven valores como la convivencia y la solidaridad.

En la tercera sala, conocida como "Teatro de la imaginación", se exponen distintas animaciones y videoclips basados principalmente en narraciones orales que cuentan aspectos de la vida cotidiana de pueblos y culturas. Los contenidos son diversos y hacen posible que los usuarios seleccionen temas de interés conforme al carácter aleatorio que ofrece la interfaz. Y la última sala, a la cual se le asignó el nombre "Palabras al aire", también emplea animaciones para que los usuarios se entretengan y a la vez aprecien los contenidos en audio. Su propósito es mantener al usuario en contacto con los contenidos, especialmente sonoros, mientras que se distrae con los juegos ya mencionados. Luego de realizar el ACDMM para resolver el objetivo del estudio, se identificaron dos categorías emergentes en los resultados: 
Contenidos digitales

para niños
Contenidos digitales para niños | Revista Virtual

Universidad Católica del Norte, 64, 119-150

ISSN: 0124-5821 (En línea)

\section{Diversidad en la multimodalidad: entre la significación y los estereotipos}

Una de las salas con mayor riqueza verbal y sonora es "Mi casa en el árbol", un espacio con una interfaz gráfica atractiva para el usuario, que explicita las opciones de selección de manera clara y que propone un ambiente gratificante para los niños, dada la escena que expone: una casa que, al parecer permite jugar y divertirse, construida alrededor del tronco de un árbol. La imagen suele estar reforzada por lo verbal y el mensaje lingüístico. Un contenido destacable de la sala en mención es "Carrusel", el cual propone al usuario que relacione el rostro de un personaje con una emoción. No obstante, la narrativa de este contenido pierde fuerza en el desarrollo del juego, pues el procedimiento que propone el algoritmo es el mismo con cada uno de los personajes. Siempre que aparece un personaje, la aplicación repite el esquema instrucción- procedimiento (siempre se ubican dos elementos en la cara del personaje) - gratificación (frase "Lo hiciste muy bien").

En esta sala es clara la intención de representar elementos de la diversidad cultural a través de cantos y karaokes que develan las riquezas simbólicas y materiales de poblaciones, como los indígenas Kamëntsá, ubicados en el Valle del Sibundoy (Putumayo), y los afrodescendientes, en este caso de la comunidad de San Basilio de Palenque (Bolívar) y de San Andrés y Providencia. Casi siempre se presentan paisajes, indumentarias, artefactos, lenguas y ritmos de las regiones Atlántica, Pacífica y Amazónica, aunque también se incluyen canciones del pueblo gitano, en los cuales aparecen niños de primera infancia como protagonistas. Dentro de los elementos de la diversidad también se destacan siete cuentos creados por niños de diferentes regiones del país, resultado de las producciones radiales del proyecto Con mi cuento encanto del Ministerio de Cultura. No obstante, en relación con la representación de los territorios de las regiones, llama la atención que estos casi siempre se utilizan como accesorios de los contenidos. En varios casos, los espacios representados no tienen relación directa con las actividades propuestas ni se convierten en referentes útiles para las interacciones de los usuarios. 


\section{Contenidos digitales} para niños
Contenidos digitales para niños | Revista Virtual

Universidad Católica del Norte, 64, 119-150

ISSN: 0124-5821 (En línea)

\section{Figura 1}

Captura de pantalla de la página de inicio del portal Maguaré (home)

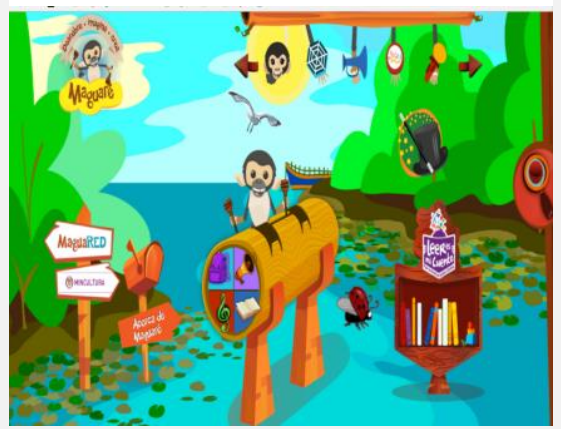

Nota: tomado de portal Maguaré.

\section{Figura 2}

Captura de pantalla de paisaje representativo de la región Amazónica colombiana (video animado "Un día en la vida de Saimiri")

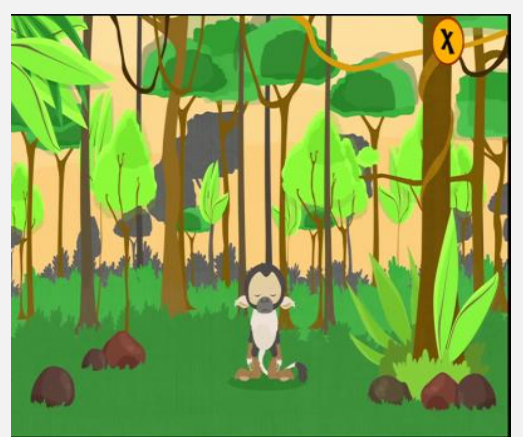

Nota: tomado de portal Maguaré. 
Contenidos digitales

para niños
Contenidos digitales para niños | Revista Virtual

Universidad Católica del Norte, 64, 119-150

ISSN: 0124-5821 (En línea)

\section{Figura 3}

Captura de pantalla de personajes del pueblo indígena Kamëntsá(Valle del Sibundoy, Putumayo) en el contenido digital "Cantos"

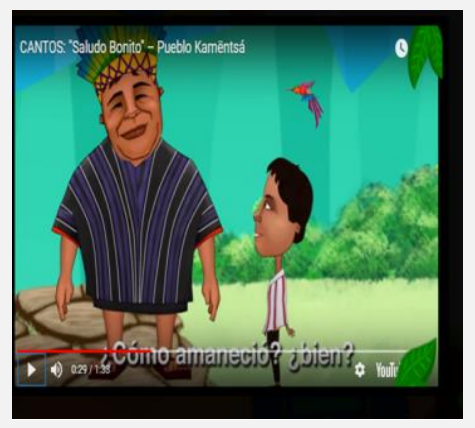

Nota: tomado de portal Maguaré.

Por otro lado, los contenidos "Pipo mi amigo imaginario", "Ana pirata" y "Guillermina y Candelario" son narrativas animadas con un alto nivel de elaboración visual. Se observa en cada caso diseños que cuidan rigurosamente la imagen, los colores, las escenas y todo lo concerniente a la animación, el paisaje sonoro y la verbalización de los personajes. Sin embargo, es importante analizar algunas funciones pragmáticas de los modos visuales en cada una de estas tres historias. En primer lugar, "Pipo" es un personaje imaginario que se le aparece a los niños y que generalmente les propone juegos, aventuras y diversión. La escala de colores, las proporciones de las imágenes y el contexto en el que se despliega la narrativa están muy bien trabajados en el diseño y la producción. Tanto "Pipo" como los demás personajes que aparecen en cada capítulo son blancos, delgados y con cabello rubio o castaño. En segundo lugar, "Ana Pirata" es una niña que se transporta con su imaginación a un mundo en el que vive aventuras como pirata, superando desafíos que surgen como consecuencia de un pirata malo que la quiere afectar. Además de tener gran imaginación, es una niña inteligente que resulta avante ante las trampas y eventualidades que surgen en cada capítulo. La narrativa visual está acompañada de gestos, movimientos de brazos y manos, y expresiones emocionales que se pueden evidenciar ante las adversidades y la felicidad de cada episodio.

Por último, los realizadores de "Guillermina y Candelario" identifican a sus personajes con la playa, las palmeras y las viviendas de palafitos del Pacífico colombiano, 


\section{Contenidos digitales}

para niños
Contenidos digitales para niños | Revista Virtual

Universidad Católica del Norte, 64, 119-150

ISSN: 0124-5821 (En línea)

así como con la alegría, la expresión verbal y el movimiento corporal. Se trata de una realización en la que sus personajes, niños afrocolombianos, pasan por diversas aventuras en su diario vivir. Guillermina y Candelario, y en general los personajes de la saga, tienen las cabezas más grandes que sus cuerpos, sus mentones son protuberantes y generalmente aparecen en la playa o en lugares exóticos del paisaje del Pacífico. También, se evidencia en lo visual el interés de sus creadores por asociar la imagen de los afros con los deportes, los bailes y la música. Esto se refuerza en el modo verbal con las voces de los personajes. Al parecer, quienes hacen las voces en la producción no son oriundos del Chocó y eventualmente intentan simular expresiones, tonos y acentos que exageran.

Como se puede apreciar en este primer tipo de resultado, si bien la interfaz y los aspectos de la composición de estos contenidos digitales son de alta calidad, estos suelen tramitar representaciones sobre la realidad social y cultural que pueden resultar confusas para los niños de primera infancia. Específicamente, en los contenidos analizados se pueden evidenciar las limitaciones de los discursos verbal y visual del portal en la tramitación de representaciones sobre la diversidad cultural, territorial y étnica, al marginar el valor del entorno natural en las actividades propuestas a los niños, así como reproducir estereotipos sobre las personas afrocolombianas que naturalizan la racialización y la exclusión de estas frente a las mayorías blanco - mestizas. Estos resultados se diferencian de lo expuesto por Chaudron et al. (2015), Lizarazo (2012) y Paudel et al. (2017), quienes sostienen que lo fundamental en este tipo de contenidos digitales es el diseño, la accesibilidad y la navegabilidad de los usuarios.

\section{Figura 4}

Captura de pantalla la serie "Pipo mi amigo imaginario"

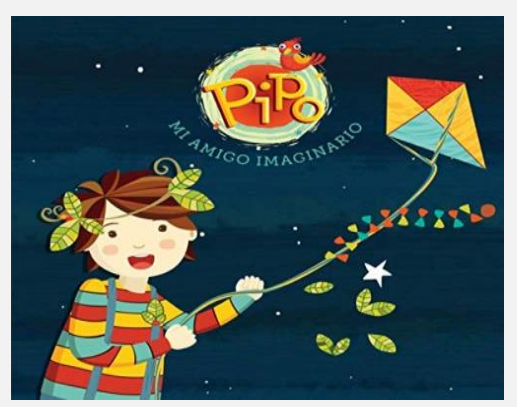

Nota: tomado de portal Maguaré. 
Contenidos digitales

para niños

\section{Figura 5}

Captura de pantalla de la serie "Guillermina y Candelario"

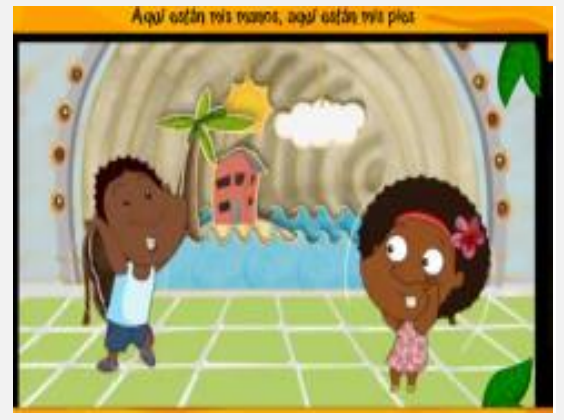

Nota: tomado de portal Maguaré.

\section{Paisajes sonoros: el reparto de lo sensible}

Caja de música es una sala constituida por una interfaz gráfica que asegura las proporciones de tridimensionalidad y organización entre los espacios y los objetos virtuales, similar a la anterior sala. La escena no corresponde a una caja de música, sino más bien a una casa o a un jardín, similar a las dimensiones de "Mi casa en el árbol". Es destacable el uso de la escala de colores lila y su complemento con el color azul aguamarina. La sala presenta ocho objetos, correspondientes a los contenidos que puede explorar el usuario. La disposición y distribución de los objetos virtuales en la interfaz gráfica evidencian menos saturación que la anterior sala y permiten hacer recorridos visuales más precisos, lo cual puede favorecer la interactividad del usuario. El modo visual de la sala es muy rico, pero vale señalar que su fortaleza está en lo verbal y lo sonoro.

A modo de ejemplo, "Camusi Camusi" es un contenido de esta sala predominantemente auditivo, en el cual los modos verbal y sonoro proponen al usuario la experimentación de sonidos, sílabas y palabras, y que logra articular, desde lo sensible, la oralidad, la expresión rítmica y el paisaje sonoro. Las experimentaciones mencionadas crean un ambiente especial de disfrute al usuario que refuerza, mediante lo verbal y lo sonoro, relaciones entre el juego, las palabras y la lectura. Por su parte, "Constelaciones Sonoras" es un contenido que promueve una vinculación directa entre imagen y paisaje sonoro, específicamente mediante escenas en las que se encuentra el firmamento, los 


\section{Contenidos digitales}

para niños
Contenidos digitales para niños | Revista Virtual

Universidad Católica del Norte, 64, 119-150

ISSN: 0124-5821 (En línea)

sonidos estelares y el propio usuario. El paisaje sonoro es cuidadosamente incorporado a la escena $y$, aunque el usuario solo debe encender un botón digital para activar audio e imagen de manera aleatoria, es una propuesta que propicia la sensibilidad, la percepción y la apreciación auditiva.

En consonancia con lo anterior, el contenido denominado "Videos musicales" es una producción de gran calidad sonora y musical. Su fortaleza está en la puesta en escena de la diversidad de expresiones musicales orientadas a los niños. Se destacan las canciones tradicionales del folclor colombiano, las rondas infantiles y géneros no convencionales para la infancia, como "rockcito". Por último, en el contenido "Cantos" se despliegan producciones musicales de gran elaboración. La puesta en escena permite la experimentación de lo sonoro y posibilita la sensibilización y el disfrute, incluso de textos sonoros desconocidos para el niño. Asimismo, "Arrullos" es un contenido que evidencia un proceso producción y postproducción riguroso en la musicalización y en los sonidos que hacen posible significar el cuidado, la acogida y el gusto por sentirse parte de un vínculo con figuras como los padres y los cuidadores de la comunidad.

\section{Figura 6}

Captura de pantalla del contenido digital Constelaciones Sonoras

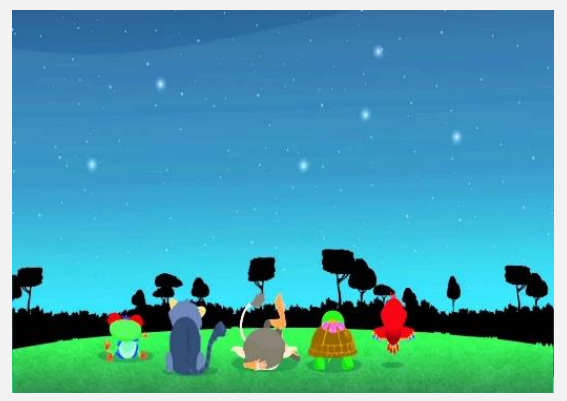

Nota: tomado de portal Maguaré. 
Contenidos digitales

para niños

\section{Figura 7}

Captura de pantalla del contenido Camusi Camusi

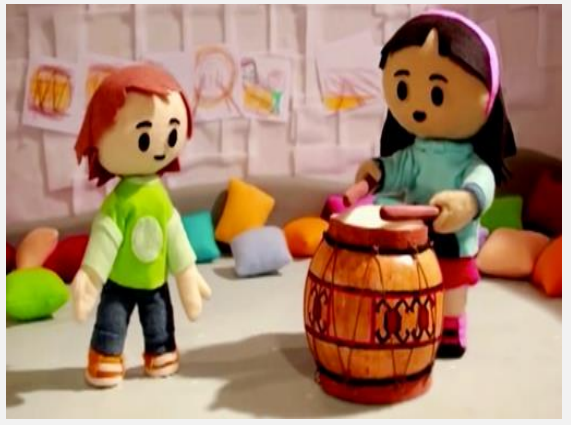

Nota: tomado de portal Maguaré.

Como se puede apreciar en estos resultados, los contenidos digitales para niños de primera infancia, tal como ocurre con Maguaré, deben no solo garantizar sistemas de signos y objetos virtuales atractivos para las audiencias, sino también formas de complementariedad e hibridación entre modos semióticos, con el fin de propiciar el goce, el aprendizaje y la significación. En este caso, los complementos y articulaciones entre lo visual, lo verbal y lo sonoro hacen posible que los mensajes, en situaciones de comunicación precisas, fomenten la sensorialidad, el afecto y la emoción en los niños. Esta mirada coincide con lo expuesto por Scolari (2008) y Díez-Gutiérrez \& Díaz-Nafría (2018), en cuanto a los aportes de los formatos hipermediales y transmediales en la configuración de otras sensibilidades y estéticas en los usuarios, pero se distancia de la perspectiva de Hiniker et al. (2016), en lo que concierne a las "rabietas" de los niños de preescolar en su exposición permanente a las pantallas y los conflictos que estas situaciones producen con pares y adultos.

\section{Discusión}

En relación con esta aproximación a los contenidos multimodales del portal Maguaré, se pueden destacar cuatro consideraciones. La primera sostiene que, a pesar de los esquemas repetitivos de algunas aplicaciones del portal, son útiles los complementos propuestos por los realizadores entre palabra verbal (mediatizada), imagen y texto 
Contenidos digitales

para niños
Contenidos digitales para niños | Revista Virtual

Universidad Católica del Norte, 64, 119-150

ISSN: 0124-5821 (En línea)

alfabético, los cuales se constituyen en repertorios semióticos que ofrecen distintas potencialidades para la comunicación y la producción de significación en el usuario. Lo verbal, mediante el mensaje hablado (narradora del portal), posibilita una comunicación directa entre la interfaz y el niño. Se trata de un modo semiótico que, al integrar elementos sonoros y visuales alrededor de lo verbal, facilita la comunicación bidireccional entre el portal y el usuario. En dicho proceso de comunicación, el carácter multimodal del contenido hace posible que la recepción sea intensamente sensorial (Manghi et al., 2014) y que la intuición se constituya en la base de la navegación en el portal

En otras palabras, el mensaje lingüístico (mediatizado) también permite al usuario integrar la imagen y la codificación textual, situación que invita a elegir opciones y tomar decisiones en el marco de una situación de comunicación digital propuesta (Scolari, 2008). Por otro lado, a diferencia de lo expuesto por Hiniker et al. (2016), quienes plantean que los usos de los contenidos digitales en los niños suelen convertirse en objeto de conflicto familiar, se puede señalar que la composición semiótica de este espacio virtual permite a los niños usar y apropiar cúmulos de información y conocimientos, a partir de diversos artefactos semióticos, entre ellos textos alfanuméricos con esquemas gráficos, mapas e imágenes visuales bidimensionales o tridimensionales que fomentan otros marcos de significación (Coenen et al., 2015; Kress \& Van Leeuwen, 2001).

Una segunda consideración de este análisis multimodal del portal tiene que ver con el reconocimiento de la diversidad y la diferencia. Como se anotó, los contenidos de varias salas muestran imágenes (especialmente cuerpos, grupos, objetos y espacios), lenguas distintas al español (especialmente indígenas y palenqueras) y paisajes sonoros, diferentes al entorno predominantemente urbanocéntrico del niño de primera infancia, los cuales se constituyen en referentes divergentes para la significación. Se trata de otras fuentes culturales de conocimiento y significación que, en muchos casos, no hacen parte de los discursos y prácticas que configuran la vida cotidiana del niño. Esta manera de mostrar la diversidad y la diferencia al usuario, a través del portal, evidencia que los referentes del mundo no son exclusivamente eurocéntricos, monoculturales ni alfabéticos. En el universo narrativo de Maguaré se presenta una realidad multicultural que comprende variados y complejos saberes, dinámicas y narrativas, los cuales dan cuenta de otras lógicas societales y culturales, que ponen a disposición del niño horizontes humanos 
Contenidos digitales

para niños
Contenidos digitales para niños | Revista Virtual

Universidad Católica del Norte, 64, 119-150

ISSN: 0124-5821 (En línea)

radicalmente distintos (tradiciones o lugares culturales - topoi- diferentes) que pueden hacer posible que los usuarios produzcan valoraciones distintas de estos otros sistemas culturales (Mejía, 2016; Santos, 2009), así sean simulados digitalmente. En suma, el carácter multimodal del portal propone puntos de encuentro al usuario entre mythos y logos, entre subjetividad y objetividad, y entre pensamiento racional y emocional.

Como tercera consideración, si bien existen varios contenidos que muestran la diversidad y la diferencia de la nación colombiana, también es claro que Maguaré cae en estereotipos, específicamente a partir de las representaciones de determinados personajes. Estereotipos que resultan problemáticos en la construcción del mundo social de los niños de primera infancia. Como se mencionó, en el entorno llamado "Mi casa en el árbol", mientras que Pipo (niño- masculino), es blanco, inteligente, equilibrado y posee una expresión verbal eficiente, y Ana es una mestiza aventurera que supera la adversidad con inteligencia e intuición, Guillermina y Candelario además de ser representados de manera asimétrica entre la cabeza y el cuerpo, evidencian que sus habilidades están asociadas a la fiesta y el juego. En suma, se evidencia una orientación cognitiva e ideológica de folclorización y racialización desde el discurso multimodal (Kress \& Van Leewen, 2001).

En consecuencia, es posible que, al comparar las tres narrativas, el niño de primera infancia construya un régimen de la mirada en el que encuentra mayor afinidad por los personajes blancos y mestizos, y en el que asuma la existencia de una suerte de diferencia estructural que naturaliza al personaje afro con el déficit y el folclor. Esta forma de discriminación positiva en lugar de promover el reconocimiento de otros valores y capacidades de las personas afro se constituye en una estrategia de racialización que ancla a estas personas en una diferencia subalternizada y marginal, inventada y narrada desde un mundo imaginado blanco-mestizo ideal (Maldonado-Torres, 2006; Masferrer, 2017). Asimismo, el portal no propone narrativas en donde interactúen los pueblos y culturas representados. Más bien, estos se presentan como comunidades particulares, separadas de la estructura social, sin capacidades para construir lo común, situación que evidencia una mirada multicultural (no intercultural) del mundo por parte de sus realizadores.

Por último, se puede señalar que el predominio del modo sonoro en algunos contenidos del portal contribuye a que los niños de primera infancia participen del reparto 
Contenidos digitales

para niños
Contenidos digitales para niños | Revista Virtual

Universidad Católica del Norte, 64, 119-150

ISSN: 0124-5821 (En línea)

de lo sensible (Rancière, 2008), esto es, un sistema de evidencias sensibles que hacen posible la ampliación de la significación y la construcción de lo común. De acuerdo con Rancière (2008), esta repartición se funda en una distribución de espacios, tiempos y formas de actividad que habilita a los actores sociales para que participen de procesos éticos, estéticos y políticos, orientados hacia la solidaridad y la intersubjetividad. Por otro lado, este reparto favorece las formas de visibilidad de las prácticas estéticas y artísticas con respecto a lo común. Lo común en este estudio es un proceso que vincula al creador y al usuario a partir de la mediación multimodal del portal. En este caso, el usuario no solo funge como receptor pasivo, sino también como co-creador (Chaudron et al., 2015; Siemens, 2007), a partir de operaciones que estimulan la percepción y la sensibilidad.

De este modo, el reparto de lo sensible en el portal Maguaré es un proceso que está a la base de la estética. Aquí la estética no se inscribe en el régimen de la mirada de lo bello o en los referentes canónicos del arte, sino en un modo de realidad que apela a lo sensible como mecanismo de encuentro con el mundo en sus dimensiones abstractas y fenoménicas. De acuerdo con Rancière (2008), el reparto de lo sensible implica su redistribución y reconfiguración, así como iniciativas que hagan posible la construcción del sentido de comunidad. Ante las ausencias y confusiones que comprenden lo común en Colombia, el portal Maguaré es una especie de mosaico de narrativas cuyas piezas pueden contribuir a construir lo común desde la diferencia cultural y el patrimonio simbólico de lo sonoro, lo visual y lo verbal. El cruce de diversas voces y experiencias hace posible la configuración de lo político, según Rancière (2008), situación que potencia otros modos de relación de los niños con los otros y con el entorno (Guerrero-Cabrera et al., 2018). En tal sentido, el universo digital de Maguaré es una oportunidad para que los niños de primera infancia se incorporen en el reparto de lo sensible. Esto tendría que ser analizado a partir de los consumos (usos y apropiaciones) de los contenidos realizados por los participantes en próximos estudios.

Tanto los resultados como el análisis de este estudio sugieren tres tipos de implicaciones prácticas. Por un lado, es importante que los decisores de la política pública de primera infancia asuman el diseño de contenidos digitales sobre la realidad social y cultural, desde una perspectiva intercultural que represente la diversidad, sin caer en exclusiones ni estereotipos, asociados con la etnia, el género, la clase social o el territorio. Por otro lado, los resultados evidencian que los diseñadores y artistas visuales deben asumir que los niños son actores sociales con capacidad de agencia, y que pueden experimentar diversos modos semióticos que contribuyan a enriquecer su 
Contenidos digitales

para niños
Contenidos digitales para niños | Revista Virtual

Universidad Católica del Norte, 64, 119-150

ISSN: 0124-5821 (En línea)

significación, a partir de mediaciones digitales que favorezcan el reconocimiento de lo diferente y su capacidad de asombro a partir de la experiencia estética. Por último, si bien los padres de familia, profesores y cuidadores deben garantizar el control parental de los niños, tal como lo establecen las normas nacionales e internacionales, este estudio demuestra que el discurso verbal, sonoro y visual de los contenidos puede favorecer la experimentación sensible de los niños y el goce de las narrativas digitales online, sin que estas experiencias se conviertan en conflictos intergeneracionales, tal como lo plantean otros estudios (Hiniker et al., 2016).

\section{Conclusiones}

Los contenidos digitales dirigidos a niños de primera infancia tienen como propósito contribuir a la educación de estas personas, aunque también ofrecer referentes para que estos se aproximen a información, conocimientos, valores y experiencias relacionados con otros grupos sociales y otros entornos naturales y culturales. Aunque se trata de un objeto de estudio reciente, este tipo de contenidos ha mostrado su potencial para la innovación educativa, la formación del talento humano y la generación de experiencias interactivas conducentes a ampliar los marcos de representación y de significación de sus usuarios. En este horizonte de reflexión, surge Maguaré, un portal digital cuyos contenidos buscan representar la diversidad y la diferencia cultural de Colombia, dirigido a niños de 3 a 6 años. De acuerdo con sus diseñadores y gestores, se espera que los usuarios alcancen nuevas comprensiones de la realidad social y cultural de la Nación por medio de este universo narrativo.

Los antecedentes evidencian que este tipo de portales han tenido un crecimiento considerable en distintos países durante los últimos veinte años. Mientras que algunos hacen énfasis en contenidos orientados hacia el juego, la música y el arte, otros buscan desarrollar habilidades relacionadas con procesos de lectura y escritura, operaciones lógicomatemáticas y estimulación temprana. Asimismo, se observa que, aunque existen diferencias importantes entre las iniciativas estatales y privadas, un común denominador de este tipo de dispositivos es la incorporación de diversos modos semióticos en los contenidos 
Contenidos digitales

para niños
Contenidos digitales para niños | Revista Virtual

Universidad Católica del Norte, 64, 119-150

ISSN: 0124-5821 (En línea)

propuestos, así como una importante tendencia hacia la interactividad, la navegabilidad, la usabilidad y la accesibilidad. Para algunos autores, estos atributos se enmarcan en una modalidad nueva de comunicación digital interactiva, en otros repertorios de consumo simbólico, así como en modalidades emergentes de interacción y creación.

Por otro lado, la primera infancia, entendida como una construcción social que surge de un entramado de discursos, políticas, conocimientos y prácticas sobre los niños de 0 a 6 años, se ha convertido en objeto de la política pública y de nuevas modalidades de atención y cuidado, en la mayoría de los países, a lo largo de las tres últimas décadas. La literatura muestra que los discursos y conocimientos enmarcados en los derechos del niño, su desarrollo integral y el retorno de la inversión se constituyen en las grandes narrativas que orientan dichas políticas en la mayoría de las sociedades. No obstante, es necesario tener en cuenta que existen otras condiciones de tipo social, político y cultural en las que se tramita la construcción de la primera infancia, entre ellas, la emergencia de culturas infantiles ligadas al mercado simbólico, otras formas de comunicación medidas por tecnologías digitales, otros modos de socialización y otras formas de sensibilidad. Este panorama genera inquietudes relacionadas con las funciones de estas formas de comunicación y consumo simbólico en el desarrollo de los niños, las características de los discursos que se incorporan en estos contenidos digitales, así como las relaciones posibles entre este tipo de discursos multimodales y el régimen de la mirada producido por sus usuarios. En relación con esta última inquietud, parece necesario problematizar qué políticas de la mirada sobre la diversidad y la diferencia propician los contenidos de Maguaré en su audiencia.

A partir del análisis crítico del discurso multimodal fue posible identificar tres tipos de hallazgos. En primer lugar, varios contenidos del portal Maguaré ofrecen repertorios semióticos diversos, en los que se integran la imagen, lo sonoro, lo verbal y el mensaje lingüístico, los cuales se constituyen en dispositivos semióticos que potencian la producción del significado en sus usuarios. Además de ofrecer al usuario alternativas para acceder a la información y el conocimiento de ciertos referentes para ampliar la mirada del otro y del mundo, por ejemplo, un canto de un pueblo indígena, un paisaje natural de algún lugar de Colombia o una narrativa fantástica que incluye al niño como protagonista, los contenidos proponen integraciones diversas de estos modos semióticos, situación que permite a los niños apropiar estas representaciones por medio de las vías sensorial, afectiva y expresiva. 


\section{Contenidos digitales}

para niños
Contenidos digitales para niños | Revista Virtual

Universidad Católica del Norte, 64, 119-150

ISSN: 0124-5821 (En línea)

En segundo lugar, los resultados muestran que, si bien varios de los contenidos analizados ofrecen a sus usuarios otras fuentes sociales y culturales para acceder al carácter diverso y multicultural de la realidad local y nacional, especialmente mediante la representación de imágenes, lenguas y paisajes no urbanocéntricos, el portal utiliza algunos estereotipos. Específicamente, algunas narrativas que representan a pueblos afrocolombianos emplean estrategias de racialización y folclorización, basadas en el déficit y la discriminación positiva. Estos estereotipos, en lugar de fomentar la valoración de la diferencia cultural, se constituyen en un obstáculo ontológico y epistémico para alcanzar nuevas comprensiones sobre el mundo social en los niños, y promueve un régimen de la mirada que asocia al otro con la subalternidad y la marginalidad. Como complemento a estos estereotipos, también llama la atención la ausencia de narrativas en el portal que muestren la interacción y la negociación cultural entre pueblos y culturas. Estas suelen mostrarse como independientes y apartadas.

Por último, se puede afirmar que el predominio del modo sonoro en los contenidos del portal es un atributo que favorece la percepción, la sensibilidad y el reparto de lo sensible. Lo sonoro se evidencia en piezas musicales, canciones, rondas, juegos y leyendas tradicionales, que se integran con lenguajes visuales, textos alfabéticos y narrativas interactivas. Este proceso de hibridación semiótica aporta no solo a la ampliación de la significación en los usuarios, sino que también propicia la experimentación de procesos éticos, estéticos y políticos, los cuales pueden llegar a promover la solidaridad, la empatía y la intersubjetividad. De acuerdo con lo expuesto, es necesario que futuros estudios analicen de qué manera las audiencias, en este caso, niños de primera infancia, usan, apropian y consumen este tipo de contenidos. También, es importante comprender qué narrativas construyen los niños en torno a las representaciones de la diversidad y de la diferencia cultural, promovidas por este tipo de contenidos. Por último, se requiere profundizar qué prácticas y procesos desarrollan los usuarios, por medio de estas interfaces, como expresión de las culturas infantiles emergentes en las sociedades contemporáneas. 


\section{Contenidos digitales}

para niños
Contenidos digitales para niños | Revista Virtual

Universidad Católica del Norte, 64, 119-150

ISSN: 0124-5821 (En línea)

\section{Referencias}

Amador-Baquiro, J. C. (2018). Educación interactiva a través de narrativas transmedia: posibilidades en la escuela. Magis, Revista Internacional de Investigación en Educación, 10(18), 77-94. https://revistas.javeriana.edu.co/index.php/MAGIS/article/view/21781

Aparici, R., y Silva, M. (2012). Pedagogía de la interactividad. Comunicar, 19(38), 51-58. https://www.revistacomunicar.com/index.php?contenido=detalles\&numero $=38 \&$ articulo=3 8-2012-07

Arango, S. (2014). Cuentos Infantiles Interactivos: herramientas lúdico-didácticas para niños entre 3 y 5 años. Caso de estudio: PlayTales (Trabajo de grado de Maestría). Universidad de Palermo.

https://fido.palermo.edu/servicios_dyc/tesis_maestria/detalle_proyecto.php?id_proyecto=32 $\underline{77}$

Asamblea General de las Naciones Unidas. (1989). Convención Internacional de los Derechos del Niño (1989). https://www.ohchr.org/sp/professionalinterest/pages/crc.aspx

Barragán, D., \& Amador, J. C (2020). Appropriation of ICT in the educational field: approach to public policy in Colombia years 2000-2019. Digital Education Review, (37), 109- 129. https://revistes.ub.edu/index.php/der/article/view/30609

Benítez, R. (2013). Utilización de tecnologías de la información y comunicación por niños y niñas, en ámbitos formales e informales (Trabajo de grado de pregrado). Universidad de la República. $\quad$ https://slidex.tips/download/utilizacion-de-tecnologias-de-la-informacion-ycomunicacion-por-nios-y-nias-en-a

Buckingham, D. (2012). Repensando el niño-consumidor: nuevas prácticas, nuevos paradigmas. $\begin{array}{lllll}\text { Comunicação, } & \text { Mídia } & \text { Consumo, } & 9(25), & \text { 43-72. }\end{array}$ https://www.researchgate.net/publication/277984625_Repensando_a_Criancaconsumidora_Novas_Praticas_Novos_Paradigmas/fulltext/57aa038b08ae0932c96e5335/Re pensando-a-Crianca-consumidora-Novas-Praticas-Novos-Paradigmas.pdf

Buckingham, D., \& Tingstad, V. (Eds.) (2010). Childhood and Consumer Culture. Palgrave Macmillan.

Chaudron, S., Beutel, M. E., Donoso Navarrete, V., Dreier, M., Fletcher-Watson, B., Heikkilä, 


\section{Contenidos digitales}

para niños
Contenidos digitales para niños | Revista Virtual

Universidad Católica del Norte, 64, 119-150

ISSN: 0124-5821 (En línea)

A.S., \& Mascheroni, G. (2015). Young children (0-8) and digital technology. A qualitative exploratory study across seven countries. European Commission Joint Research Centre. https://bit.ly/3dgut7R

Chen, B. B., Wang, Y., Liang. J., \& Tong, L. (2016). And baby makes four: Biological and psychological changes and influential factors of firstborn's adjustment to transition to siblinghood. Advances in Sychological Science, 24(6), 863-873. http://journal.psych.ac.cn/xlkxjz/EN/10.3724/SP.J.1042.2016.00863

Coalición Internacional de Calificación por Edad -IARC-. (s.f.). IARC. https://www.globalratings.com/

Coenen. P., Erin, H., Amity, C., \& Leon, S. (2015). Mobile touch screen device use among young Australian children: First results from a national survey. Proceedings 19th Triennial Congress of the IEA. Melbourne. https://goo.gl/3LgcvW

Colectivo de Educación infantil y TIC, Universidad del Norte. (s.f.). Colectivo TIC. http://ylangylang.uninorte.edu.co:8080/redei/colectivo_tic.php

Corredor, M. Y., y Garzón, K. J. (2015). La Integración de las TIC en los Procesos de Educación Inicial como Apoyo para el Desarrollo del Pensamiento Crítico en los Niños y Niñas del Colegio Confraternidad de San Fernando, de la Ciudad Bogotá DC-Colombia. Memorias del XVI Encuentro Internacional Virtual Educa. http://recursos.portaleducoas.org/publicaciones/la-integraci-n-de-las-tic-en-los-procesos-deeducaci-n-inicial-como-apoyo-para-el

Díez-Gutiérrez, E., \& Díaz-Nafría, J. M. (2018). Ubiquitous learning ecologies for a critical cybercitizenship. Comunicar, 26(54), 9-58. https://doi.org/10.3916/C54-2018-05

Galvis, A. (2014). Las políticas TIC en los sistemas educativos de América Latina. Caso Colombia $\begin{array}{lllll}\text { Programa } & \text { TIC } & y & \text { Educación } & \text { Básica. }\end{array}$ https://www.researchgate.net/publication/317563128_Las_politicas_TIC_en_los_sistemas_ educativos_de_America_Latina_Caso_Colombia

Gardner, H., \& Davis, K. (2014). The app generation: How today's youth navigate identity, intimacy, and imagination in a digital world. Yale University Press. https://bit.ly/2KbzdMQ

Giráldez, A. (2013). Enseñar artes en un mundo digital: diez propuestas para la formación del profesorado. Revista Belo Horizonte, 3(5), 85-104. 
Contenidos digitales

para niños
Contenidos digitales para niños | Revista Virtual

Universidad Católica del Norte, 64, 119-150

ISSN: 0124-5821 (En línea)

https://es.scribd.com/document/283504768/Ensenar-artes-en-un-mundo-digital

Giraldo-Vargas, L. (2011). Las TIC integradas en el currículo de infantil. En J. Hernández-Ortega, M. Penessi-Fruscio, D. Sobrino-López y A. Vázquez-Gutiérrez (Coords.), Experiencias educativas en las aulas del siglo XXI. Innovación con TIC (pp. 29-33). Ariel y Fundación Telefónica.

Guerrero Cabrera, S. A., Colorado Orozco, D., y Castañeda Ruiz, H. N. (mayo-agosto, 2018). La formación ciudadana en niños de edad preescolar: un reto posible. Revista Virtual $\begin{array}{lllll}\text { Universidad Católica del } & \text { Norte, (54), }\end{array}$ https://revistavirtual.ucn.edu.co/index.php/RevistaUCN/article/view/989

Hayes, A. G. (2010). La composición musical como construcción: Herramientas para la creación y la difusión musical en internet. Revista Iberoamericana de Educación, (52), 109-125. https://rieoei.org/historico/documentos/rie52a06.pdf

Hernández, J. R. (2011). Efectos de la implementación de un programa de educación musical basado en las TIC sobre el aprendizaje de la música en Educación Primaria. Universidad de Alicante (Tesis de doctorado). Universidad de Alicante. https://pdfs.semanticscholar.org/ad5d/194f1ae970b337073704781a1b2f6fee4023.pdf

Hiniker, A., Suh, H., Cao, S., \& Kientz, J. A. (2016). Screen time tantrums: How families manage screen media experiences for toddlers and preschoolers. Computer Supported Parenting, 648-660. http://faculty.washington.edu/alexisr/ScreenTimeTantrums.pdf

Johansson, B. (2010). Subjectivities of the child consumer: beings and becomings. En D. Buckingham \& V. Tingstad (Eds.), Childhood and Consumer Culture. Palgrave Macmillan

Kelly, V. (2016). Primera infancia frente a las pantallas: de fenómeno social a asunto del Estado. Sistema de Información de Tendencias Educativas en América Latina -Siteal-, Organización de los Estados Iberoamericanos para la Educación, la Ciencia y la Cultura-OEI-, UNESCO, Instituto Internacional de Planeamiento de la Educación -IPE-, UNESCO Buenos Aires. https://cerlalc.org/publicaciones/primera-infancia-frente-a-las-pantallas-de-fenomenosocial-a-asunto-de-estado/

Kress, G., \& Van Leeuwen, T. (2001). Multimodal discourse: The modes and media of contemporary communication. Arnold.

Lévy, P. (2007). Cibercultura. La cultura de la sociedad digital. Anthropos. 


\section{Contenidos digftales}

para niños
Contenidos digitales para niños | Revista Virtual

Universidad Católica del Norte, 64, 119-150

ISSN: 0124-5821 (En línea)

Livingstone, S. (2006). Children's privacy online. En: R. Kraut, M. Brynin y S. Kiesler (Eds.), Computers, Phones and the Internet (pp. 145-167). Oxford University Press.

Lizarazo, M. (2012). ¿Cómo crear contenidos con niños y niñas? Propuesta metodológica de cocreación contextualizada de hipermedia en educación no formal para la primera infancia (Trabajo de grado de maestría). Universidad Nacional de Colombia. http://www.bdigital.unal.edu.co/8569/1/4868124.2012.pdf

Llobet, V. (2014). La producción de la categoría "niño-sujeto-de-derechos" y el discurso psi en las políticas sociales en Argentina. Una reflexión sobre el proceso de transición institucional. En Autor (Ed.), Pensar la infancia desde América Latina. Un estado de la cuestión (pp. 209235). Clacso.

Maldonado-Torres. N. (2006). Sobre la colonialidad del ser: contribuciones al desarrollo de un concepto. En S. Castro-Gómez y R. Grosfoguel (Eds.), El giro decolonial, Reflexiones para una diversidad epistémica más allá del capitalismo global. Siglo del Hombre Editores, Universidad Javeriana, IESCO.

Manghi, D., Badillo, C., y Villacura, P. (2014). Alfabetización semiótica en clases de historia, estrategias de mediación desde un enfoque multimodal. Revista Perfiles Educativos, 36(146), 63-79. https://www.sciencedirect.com/science/article/pii/S018526981470128X

Manovich, L. (2001). El lenguaje de los nuevos medios de comunicación. La imagen en la era digital. Paidós.

Martín-Barbero, J. (2003). De los medios a las mediaciones. Comunicación, cultura y hegemonía. Convenio Andrés Bello.

Masferrer, C. (2017). El racismo y la representación social de lo negro entre niños de pueblos afromexicanos. Revista Antropologías del Sur, 4(8), 169-189. http://revistas.academia.cl/index.php/rantros/article/view/762

Mejía, M. (2016). Diálogo-confrontación de saberes y negociación cultural: ejes de las pedagogías de la educación popular: una construcción desde el sur. Educar em Revista, (61), 37-53. https://revistas.pedagogica.edu.co/index.php/PYS/article/view/3866

Mustard, F. (2003). ¿Qué es el desarrollo infantil? Primera Infancia y Desarrollo. El desafío de la década. Center of the Developing Child at Harvard University. 
Contenidos digitales

para niños
Contenidos digitales para niños | Revista Virtual

Universidad Católica del Norte, 64, 119-150

ISSN: 0124-5821 (En línea)

Organización de Estados Iberoamericanos. (2000). Declaración del Simposio Mundial de Educación Parvularia o Inicial. «Una educación inicial para el siglo XXI». Revista Iberoamericana De Educación, 22, 157-160. https://doi.org/10.35362/rie2201026

Paudel, S., Jancey, J., Subedi, N., \& Leavy, J. (2017). Correlates of mobile screen media use among children aged 0-8: A systematic review. BMJ Open, 7(10). https://bmjopen.bmj.com/content/bmjopen/7/10/e014585.full.pdf

Ramos, I. B., y González, M. J. A. (2012). Formación continua del profesorado en TIC: un estudio de caso. Memorias del I Congreso Virtual Internacional sobre Innovación. https://rio.upo.es/xmlui/handle/10433/5642

Rancière, J. (2008). El espectador emancipado. Manantial.

Rodríguez Illera, J.L., Escofet, A. y Azzato, M. (2004). Un sistema abierto para la creación de contenidos educativos digitales. RED. Revista de Educación a Distancia, (4). http://www.um.es/ead/red/M4/

Romero, Y. S. (2012). Producción de televisión educativa para la primera infancia: una propuesta para potenciar el desarrollo de los niños y niñas colombianos. Memorias I Congreso Virtual Internacional sobre Innovación Pedagógica y Praxis Educativa INNOVAGOGÍA 2012. https://www.academia.edu/11462208/la_producci\%c3\%93n_de_televisi\%c3\%93n_educati va_para_la_primera_infancia_una_propuesta_para_potenciar_el_desarrollo_de_los_ni\%c3 \%910s_y_ni\%c3\%91as_colombianos

Runge-Peña, A. (2016). El cuarto infantil como espacio de relaciones intergeneracionales. En M. Passeggi, E. Cunico Furnaletto y R. Domingos da Palma (Orgs.), Pesquisa (auto) biográfica, infancias, escola e diálogos intergeneracionais. Editora CRV.

Santos, B. (2009). Una epistemología del sur: la reinvención del conocimiento y la emancipación social. Siglo XXI-CLACSO.

Scolari, C. (2008). Hipermediaciones. Elementos para una teoría de la comunicación digital interactiva. Gedisa.

Siemens, G. (2007). Situating connectivism. http://ltc.umanitoba.ca/wiki/Situating_Connectivism Solomon-Moore, E., Matthews, J., Reid, T., Toumpakari, Z., J. Sebire, S., Thompson, J. L., Lawlor, D. A. \& Jago, R. (2018). Examining the challenges posed to parents by the contemporary screen environments of children: a qualitative investigation. Pediatrics, 18(129). 


\section{Contenidos digitales}

para niños
Contenidos digitales para niños | Revista Virtual

Universidad Católica del Norte, 64, 119-150

ISSN: 0124-5821 (En línea)

https://doi.org/10.1186/s12887-018-1106-y

Thomson, K., Jaeger, P., Greene, N., Subramanian, M., \& Bertot, J. (2014). Digital Literacy and Digital Inclusion. Rowman \& Littlefield.

UNESCO. (1990). Declaración mundial sobre educación para todos. Unesco.

https://www.humanium.org/es/declaracion-mundial-sobre-educacion-para-todos-

$\underline{\text { satisfaccion-de-las-necesidades-basicas-de-aprendizaje/ }}$

UNESCO. (2000). Foro Mundial sobre Educación. Unesco.

https://unesdoc.unesco.org/ark:/48223/pf0000121117_spa

Vallejo, M. L. (2001). Una Gran Aventura por Colombia. Planteamiento conceptual de un material multimedia interactivo y didáctico de cinco ritmos tradicionales colombianos para niños de 4 a 7 años de edad (Trabajo de grado de pregrado). Universidad del Valle. http://bibliotecadigital.univalle.edu.co/bitstream/10893/4092/4/CB-0441143.pdf

Vasilachis, I. (2007). Estrategias de investigación cualitativa. Gedisa.

Winocur, R. (2009). Robinson Crusoe ya tiene celular. La conexión como espacio de control de la incertidumbre. Siglo XXI. 